\title{
Closed Reduction of Bilateral Posterior Shoulder Dislocation with Medium Impression Defect of the Humeral Head: A Case Report and Review of Its Treatment
}

\author{
Soorena Rezazadeh and Amir Reza Vosoughi \\ Research Center for Bone \& Joint Diseases, Chamran Hospital, Shiraz University of Medical Sciences, \\ Shiraz 7194815644, Iran \\ Correspondence should be addressed to Amir Reza Vosoughi, vosoughiar@hotmail.com \\ Received 29 July 2011; Revised 19 September 2011; Accepted 19 September 2011 \\ Academic Editor: Edward V. Craig \\ Copyright (C) 2011 S. Rezazadeh and A. R. Vosoughi. This is an open access article distributed under the Creative Commons \\ Attribution License, which permits unrestricted use, distribution, and reproduction in any medium, provided the original work is \\ properly cited. \\ Bilateral dislocation of the shoulder is a rare injury. The main causes are electrical shock, extreme trauma, and epilepsy. A 25- \\ year-old athletic-body man had sustained bilateral shoulder pain and restricted external rotation following electrical shock for five \\ days. Although articular surface damage was about $50 \%$ in the right side and $30 \%$ in the left, it could be managed successfully by \\ close reduction without pinning. During one-year follow-up, no recurrent dislocation or limitation of motion was seen. Closed \\ management of medium size defect of the humeral head after posterior dislocation can be performed in cooperative and especially \\ muscular patients.
}

\section{Introduction}

Pure posterior dislocation of the shoulder, a commonly missed injury, accounts for about 1 to $4.7 \%$ of all shoulder dislocations. Triple "E" syndrome shows three major causes of this entity, epilepsy, electrical shock, and extreme trauma [1]. Bilateral posterior shoulder dislocation, seen in $5 \%$ of all posterior dislocations, is the result of seizure attack in $50 \%$ of cases. Convulsive seizure will be raised to $90 \%$ of etiologies if fracture occurs concomitantly [2-4]. Electrical shock accounts for less than $5 \%$ of bilateral posterior shoulder dislocation [1].

Dislocation duration and size of the articular head defect are the major factors in determining treatment plan [58]. Acute dislocation (less than 3 weeks from the injury) and small defect up to $25 \%$ of the humeral head articular surface can be treated by closed or open reduction $[5,6]$. The presented case is the first with bilateral medium size articular defect $(25 \%$ to $50 \%)$ which was treated by closed reduction and casting.

\section{Case Report}

A 25-year-old athletic-body gentleman presented with significant pain in both shoulders and inability to do daily activities for 5 days after electrical shock. Immediately after electricity-induced trauma, he had been transferred to a major trauma center. Primary cares had been given. He had been visited by an emergency medicine physician. Routine anteroposterior radiograph of the shoulders (Figure 1) was misinterpreted. Then, he had been discharged with analgesic and arm sling. Five days later, he referred to the senior author with pain and restriction of bilateral shoulder movements. In physical exam, posterior positions of the humeral heads were not palpable due to his muscular body. The patient had fixed both upper limbs in adduction and internal rotation. Passive and active external rotation was blocked and very painful. Moreover, the patient did not permit passive abduction and forward flexion of more than 45 degrees. Neurovascular functions of both sides were normal. Bilateral shoulder posterior dislocation was suspected and CT scan was requested 


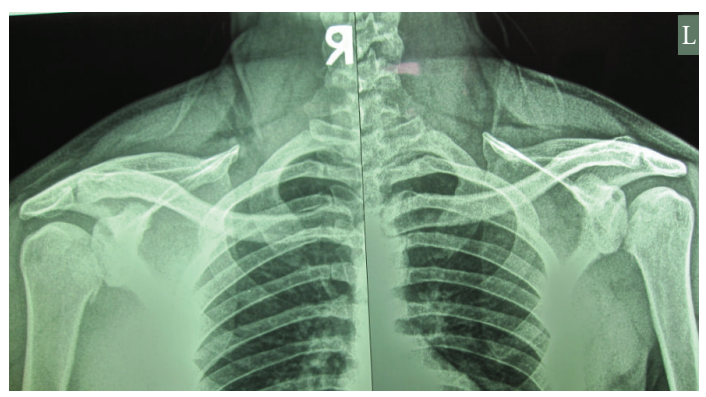

Figure 1: Anteroposterior X-ray shows bilateral posterior dislocation difficulty with absence of normal half-moon sign.

(Figure 2). It showed bilateral posterior dislocation associated with articular surface defect of $50 \%$ in the right side and $30 \%$ in the left. He underwent closed reduction under general anesthesia after taking consent. Left side reduction was stable but the right was unstable in internal rotation. Bilateral shoulder spica cast was applied in 20 degrees of abduction and 15 degrees of external rotation.

After six weeks, the cast was discarded. Stability of joints was well. Rehabilitation program including active and passive range of motion and deltoid and rotator cuff strengthening exercises were begun. As he was a professional athlete in weight lifting, he continued exercises for one year. After one-year follow-up, he had bilateral stable joint with full range of motion without any history of dislocation. CT scan (Figure 3) showed bilateral small defect in anteromedial part of the humeral heads which is more in the left side.

\section{Discussion}

Posterior shoulder dislocation is a rare injury due to very strong soft tissues behind the joint. Most are seen following tonic-clonic seizures $[3,4,6,9]$. Electrical shock and trauma especially in emotionally disabled patients are the other causes [10]. Posterior shoulder dislocations are common unrecognized injury. Hawkins et al. explained delay in diagnosis by an average of one year in $75 \%$ of cases. One of the main reasons is lack of taking axillary or lateral "Y" scapular views. Also they showed true diagnosis in all patients with axillary view [11]. Although several findings including light bulb sign, rim sign, trough line sign, absence of normal halfmoon sign, vacant glenoid sign, and Mouzopoulos sign in anteroposterior view were described, usually no one is seen $[5,12]$. Moreover, the axillary view is difficult to take due to painful abduction position. So, CT scan is a useful modality, not only for recognizing the posterior dislocation but also for determining the size of articular surface defect and associated fractures [13].

Treatment depends on patient age, duration of dislocation, extent of damage to articular surface, and patient demands and level of activity [5-7, 14, 15]. In acute cases (less than 3 weeks), closed reduction should be attempted if the articular surface defect is small (up to 25\%). Medium size defect of $25 \%$ to $50 \%$ usually needs reconstruction, lesser tuberosity transfer, or rotational osteotomy. Large defect

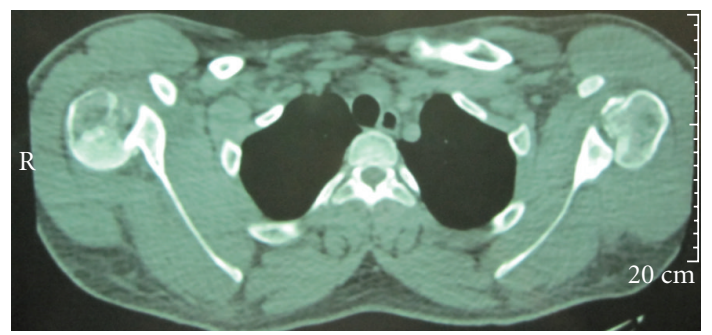

FIgURE 2: Axial CT scan view reveals bilateral shoulder posterior dislocation with medium defect of articular surfaces $(50 \%$ in the right, $30 \%$ in the left).

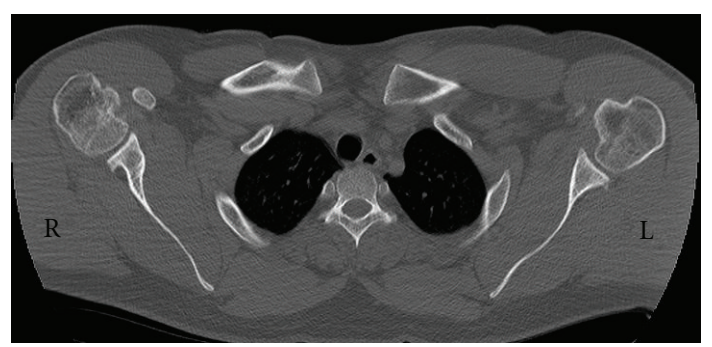

Figure 3: One-year follow-up axial CT scan view shows bilateral small defect (less than 25\%) in anteromedial part of the humeral head. These defects are less than what was seen in Figure 2.

(more than 50\%) should be managed by shoulder arthroplasty as described in the algorithm of Figure $4[6,11,15-$ 17].

The presented case had articular impression defect of $50 \%$ in the right humeral head and 30\% in the left side. We decided to reduce it closely, because he was a muscular cooperative young man. He vigorously continued strengthening exercises of the shoulder girdle muscles during followup period. He did not have any history of instability or limitation of motion.

Treatment of patients with delayed diagnosis (more than 3 weeks) depends on viability of humeral head, demand of patient, other comorbidities, and duration of dislocation. If head osteonecrosis is seen or it is diagnosed after 6 months, shoulder replacement is the modality of choice [16-18]. When the head is viable, open reduction and soft tissue release are logical. Other procedures such as humeral osteotomy, McLaughlin procedure, autograft or allograft reconstruction of the reverse Hill-Sachs lesion $[5,15,16]$ may be inevitable. Nonoperative treatment, supervised neglect, is accepted in patients with medical high risk for surgery and uncontrollable seizure disease and elderly patients with limited demand and normal motion of contralateral glenohumeral joint $[5,15]$.

In conclusion, posterior dislocation of the shoulder is a usual serious misdiagnosis. The best way for preventing unrecognized cases is suspicious in patients with pain and limitation of external rotation particularly if they have history of seizure, electrical shock, or significant trauma. CT scan can help to diagnose it early. Closed reduction of posterior shoulder dislocation in cases with medium defect in 


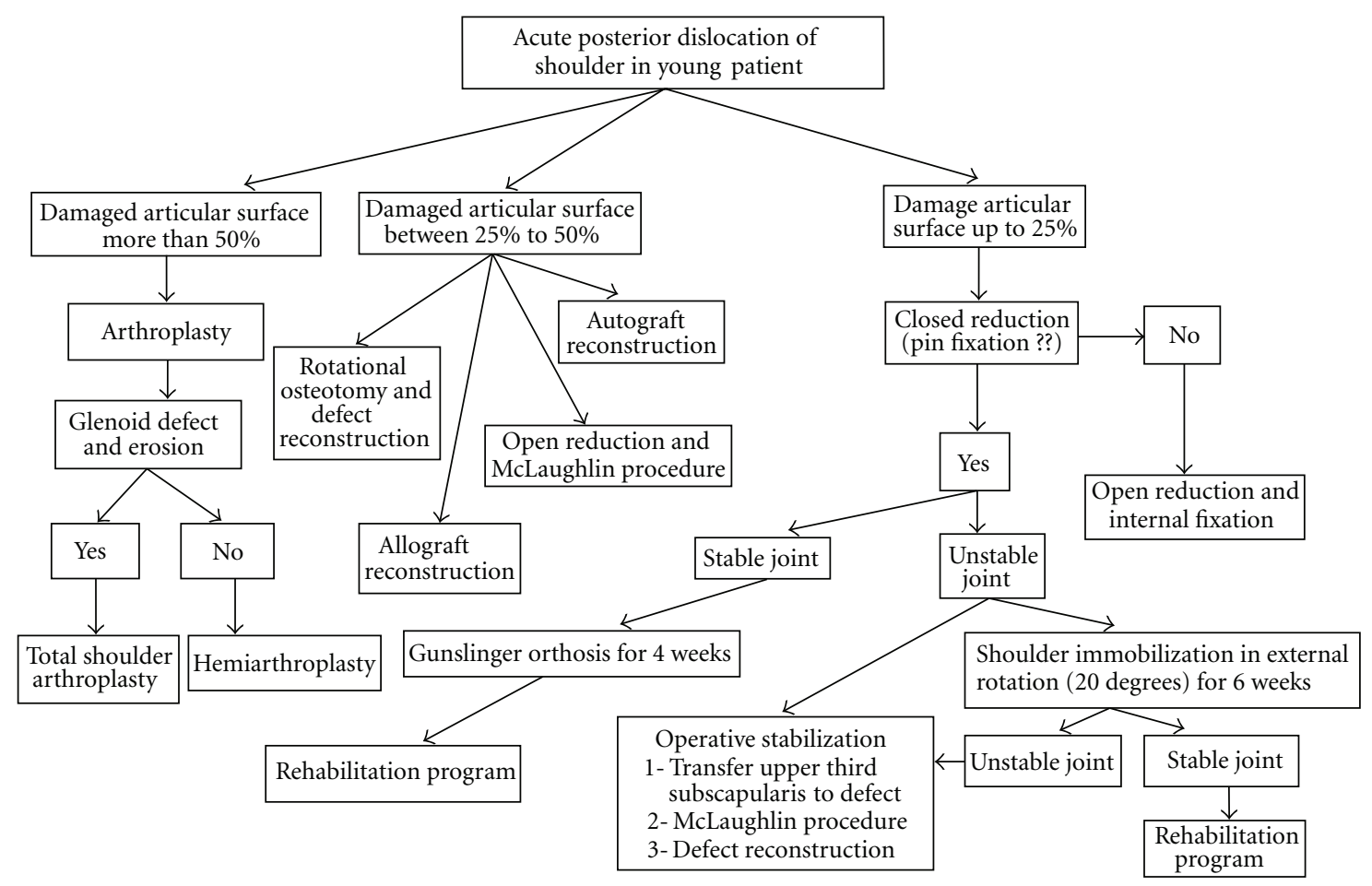

FIGURE 4: Algorithm of management of acute posterior shoulder dislocation in young patient.

the humeral head articular surface is a suitable strategy for cooperative athlete-body patients.

\section{Conflict of Interests}

The authers have no conflict of interests to declare.

\section{Acknowledgment}

The Study was carried out in Research Center for Bone \& Joint Diseases, Chamran Hospital, Shiraz University of Medical Sciences, Shiraz, Iran.

\section{References}

[1] M. Brackstone, S. D. Patterson, and A. Kertesz, "Triple "E" syndrome: bilateral locked posterior fracture dislocation of the shoulders," Neurology, vol. 56, no. 10, pp. 1403-1404, 2001.

[2] T. M. Clough and R. S. Bale, "Bilateral posterior shoulder dislocation: the importance of the axillary radiographic view," European Journal of Emergency Medicine, vol. 8, no. 2, pp. 161$163,2001$.

[3] T. D. W. Alta and W. J. Willems, "Bilateral posterior fracturedislocation of the shoulder managed by allograft reconstruction of the segmental defect: report of two cases," European Journal of Orthopaedic Surgery and Traumatology, vol. 18, no. 5, pp. 381-385, 2008.

[4] M. E. Betz and S. J. Traub, "Bilateral posterior shoulder dislocations following seizure," Internal and Emergency Medicine, vol. 2, no. 1, pp. 63-65, 2007.
[5] N. Cicak, "Posterior dislocation of the shoulder," Journal of Bone and Joint Surgery-Series B, vol. 86, no. 3, pp. 324-332, 2004.

[6] M. I. Iosifidis, I. Giannoulis, S. Traios, and G. Giantsis, "Simultaneous bilateral posterior dislocation of the shoulder: diagnostic problems and management-a case report," Knee Surgery, Sports Traumatology, Arthroscopy, vol. 14, no. 8, pp. 766-770, 2006.

[7] A. Ivkovic, I. Boric, and N. Cicak, "One-stage operation for locked bilateral posterior dislocation of the shoulder," Journal of Bone and Joint Surgery - Series B, vol. 89, no. 6, pp. 825-828, 2007.

[8] A. Delcogliano, A. Caporaso, S. Chiossi, A. Menghi, M. Cillo, and M. Delcogliano, "Surgical management of chronic, unreduced posterior dislocation of the shoulder," Knee Surgery, Sports Traumatology, Arthroscopy, vol. 13, no. 2, pp. 151-155, 2005.

[9] F. R. Hashmi, M. Pugh, and S. Bryan, "Simultaneous bilateral posterior dislocation of shoulder," American Journal of Emergency Medicine, vol. 20, no. 2, pp. 127-128, 2002.

[10] M. Carew-McColl, "Bilateral shoulder dislocations caused by electric shock," British Journal of Clinical Practice, vol. 34, no. 8-9, pp. 251-254, 1980.

[11] R. J. Hawkins, C. S. Neer, R. M. Pianta, and F. X. Mendoza, "Locked posterior dislocation of the shoulder," Journal of Bone and Joint Surgery - Series A, vol. 69, no. 1, pp. 9-18, 1987.

[12] G. Mouzopoulos, "The "Mouzopoulos" sign: a radiographic sign of posterior shoulder dislocation," Emergency Radiology, vol. 17, no. 4, pp. 317-320, 2010.

[13] G. Aparicio, E. Calvo, L. Bonilla, L. Espejo, and R. Box, "Neglected traumatic posterior dislocations of the shoulder: controversies on indications for treatment and new CT scan findings," Journal of Orthopaedic Science, vol. 5, no. 1, pp. 3742, 2000. 
[14] C. J. Hsu, H. C. Hsu, Y. C. Fong, C. C. Kuo, and D. Y. Wang, "Bilateral locked posterior fracture-dislocation of the shoulders due to electric shock," Mid-Taiwan Journal of Medicine, vol. 11, no. 2, pp. 132-137, 2006.

[15] Y. W. Kwon, K. J. Kulwicki, and J. D. Zuckerman, "Glenohumeral joint sublaxation, dislocation, and instability," in Rockwood and Green's Fractures in Adults, R. W. Bucholz, C. M. Court-Brown, J. D. Heckman, and P. Tornetta, Eds., pp. 1165-1209, Lippincott Williams \& Wilkins, Philadelphia, Pa, USA, 2010.

[16] R. Claro, R. Sousa, M. Massada, J. Ramos, and J. M. Lourenço, "Bilateral posterior fracture-dislocation of the shoulder: report of two cases," International Journal of Shoulder Surgery, vol. 3, no. 2, pp. 41-45, 2009.

[17] C. Copuroglu, B. Aykac, B. Tuncer, M. Ozcan, and E. Yalniz, "Simultaneous occurrence of acute posterior shoulder dislocation and posterior shoulder-fracture dislocation after epileptic seizure," International Journal of Shoulder Surgery, vol. 3, no. 2, pp. 49-51, 2009.

[18] G. Walch, P. Boileau, B. Martin, and H. Dejour, "Unreduced posterior luxations and fractures-luxations of the shoulder. Apropos of 30 cases," Revue de Chirurgie Orthopedique et Reparatrice de l'Appareil Moteur, vol. 76, no. 8, pp. 546-558, 1990. 


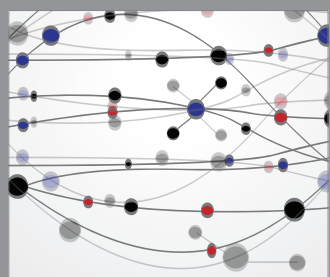

The Scientific World Journal
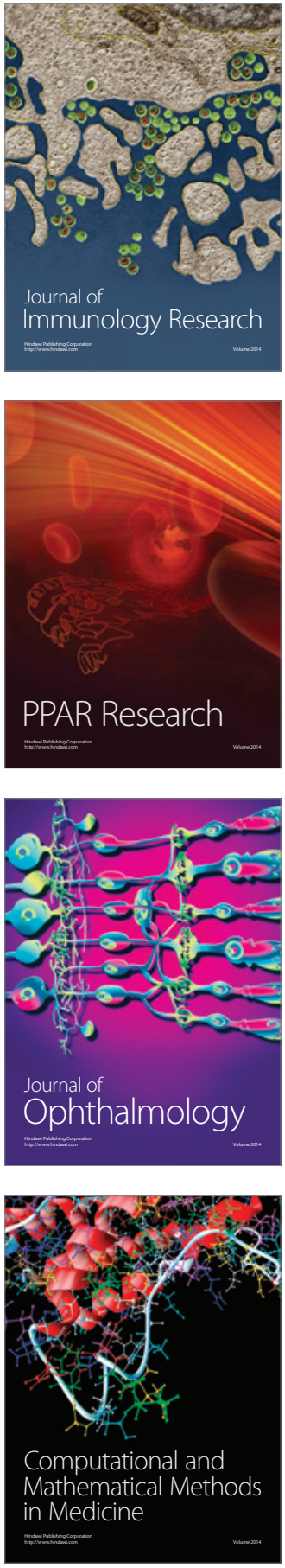

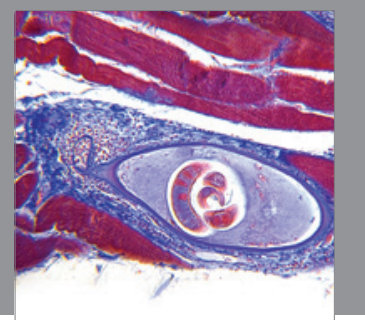

Gastroenterology

Research and Practice
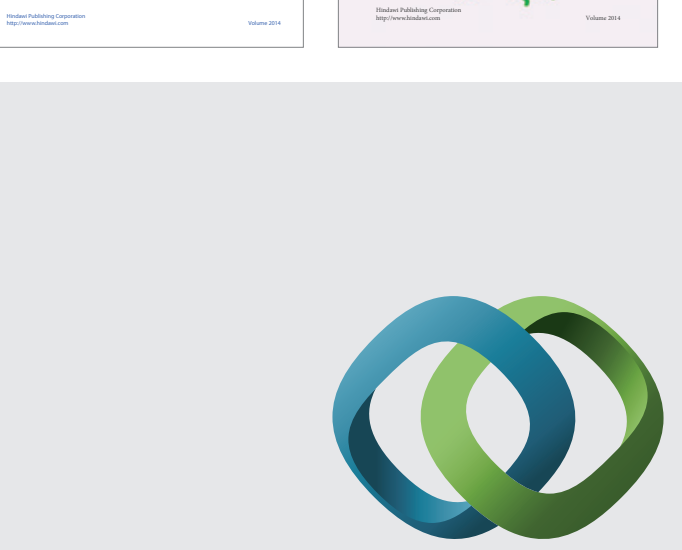

\section{Hindawi}

Submit your manuscripts at

http://www.hindawi.com
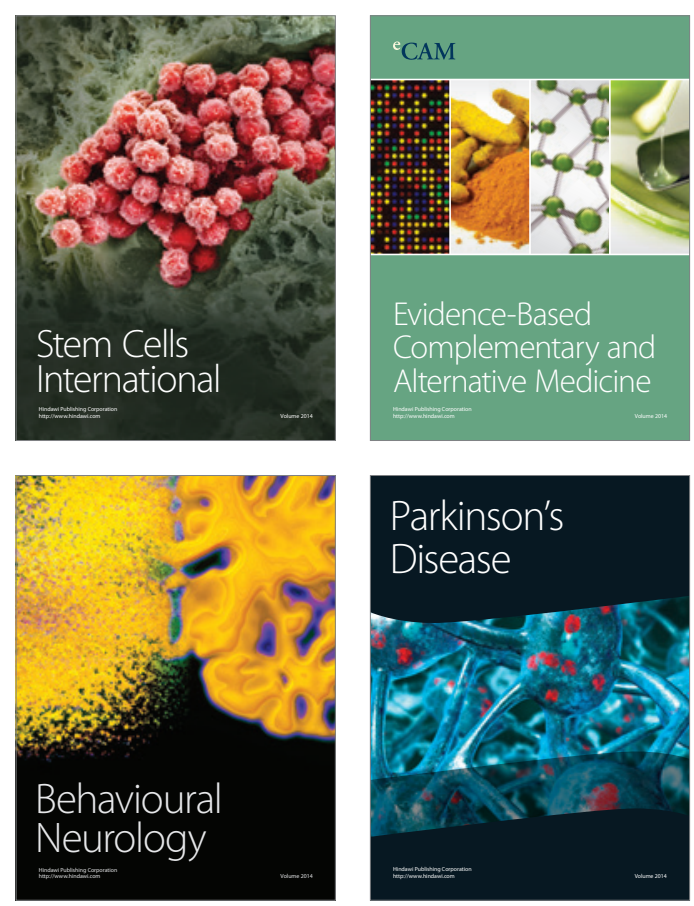

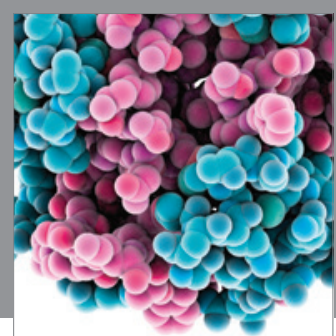

Journal of
Diabetes Research

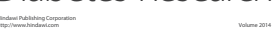

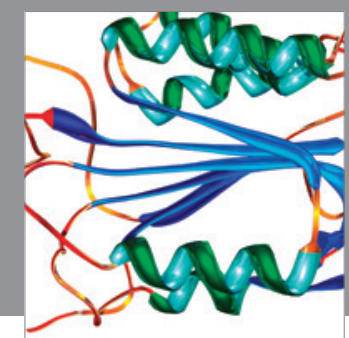

Disease Markers
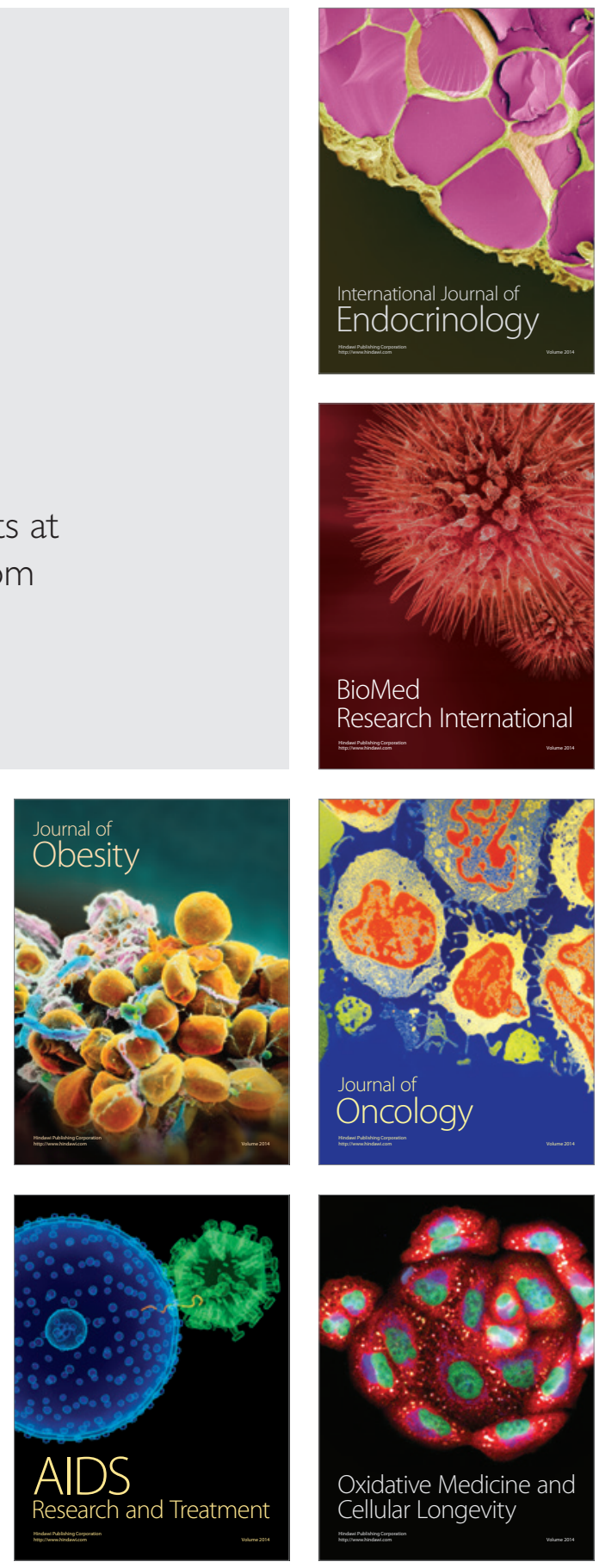